OPEN ACCESS

Edited by:

Yann Gohon,

AgroParisTech Institut

des Sciences et Industries

du Vivant et de L'environnement,

France

Reviewed by:

Mirco Dindo,

University of Perugia, Italy

Vivek Shankar Bharadwaj,

National Renewable Energy Laboratory (DOE), United States

*Correspondence: Mark A. Saper saper@umich.edu

Specialty section: This article was submitted to Plant Proteomics,

a section of the journal

Frontiers in Plant Science

Received: 11 July 2019

Accepted: 04 September 2019

Published: 11 October 2019

Citation:

Liepman AH, Vijayalakshmi J, Peisach D, Hulsebus B, Olsen LJ and Saper MA (2019) Crystal Structure Of Photorespiratory Alanine:Glyoxylate Aminotransferase 1 (AGT1) From Arabidopsis thaliana.

Front. Plant Sci. 10:1229. doi: 10.3389/fp/s.2019.01229

\section{Crystal Structure Of Photorespiratory Alanine:Glyoxylate Aminotransferase 1 (AGT1) From Arabidopsis thaliana}

\author{
Aaron H. Liepman ${ }^{1}$, J. Vijayalakshmi ${ }^{2}$, Daniel Peisach ${ }^{2}$, Brian Hulsebus ${ }^{2}$, Laura J. Olsen ${ }^{3}$, \\ and Mark A. Saper ${ }^{2 *}$ \\ ${ }^{1}$ Biology Department, Eastern Michigan University, Ypsilanti, MI, United States, ${ }^{2}$ Department of Biological Chemistry and \\ LSA Biophysics Program, University of Michigan, Ann Arbor, MI, United States, ${ }^{3}$ Department of Molecular, Cellular, and \\ Developmental Biology, University of Michigan, Ann Arbor, MI, United States
}

Photorespiration is an energetically costly metabolic pathway for the recycling of phosphoglycolate produced by the oxygenase activity of ribulose-1,5-bisphosphate carboxylase/oxygenase (RUBISCO) to phosphoglycerate. Arabidopsis alanine:glyoxylate aminotransferase 1 (AGT1) is a peroxisomal aminotransferase with a central role in photorespiration. This enzyme catalyzes various aminotransferase reactions, including serine:glyoxylate, alanine:glyoxylate, and asparagine:glyoxylate transaminations. To better understand structural features that govern the specificity of this enzyme, its crystal structures in the native form (2.2- $\AA$ resolution) and in the presence of L-serine $(2.1-\AA$ resolution) were solved. The structures confirm that this enzyme is dimeric, in agreement with studies of the active enzyme in solution. In the crystal, another dimer related by noncrystallographic symmetry makes close interactions to form a tetramer mediated in part by an extra carboxylterminal helix conserved in plant homologs of AGT1. Pyridoxal 5'-phosphate (PLP) is bound at the active site but is not held in place by covalent interactions. Residues Tyr35' and Arg36', entering the active site from the other subunits in the dimer, mediate interactions between AGT and L-serine when used as a substrate. In comparison, AGT1 from humans and AGT1 from Anabaena lack these two residues and instead position a tyrosine ring into the binding site, which accounts for their preference for L-alanine instead of L-serine. The structure also rationalizes the phenotype of the sat mutant, Pro251 to Leu, which likely affects the dimer interface near the catalytic site. This structural model of AGT1 provides valuable new information about this protein that may enable improvements to the efficiency of photorespiration.

Keywords: dimer, tetramer, substrate specificity, PLP, sat mutant, serine:glyoxylate aminotransferase, photorespiration

\section{INTRODUCTION}

The enzyme ribulose-1,5-bisphosphate carboxylase/oxygenase (RUBISCO) catalyzes most carbon fixation by photosynthetic organisms (Peterhansel et al., 2010). In current atmospheric conditions, it is estimated that the ratio of carboxylation:oxygenation of ribulose-1,5-bisphosphate (RuBP) by RUBISCO is approximately 3:1 (Sharkey, 1988); however, oxygenation becomes more pronounced at higher temperatures and in dry conditions (Peterhansel et al., 2010). When RUBISCO catalyzes carboxylation of RuBP, two molecules of 3-phosphoglycerate (PGA) are formed, and these PGA molecules enter the Calvin cycle to form sugars. When RUBISCO instead oxygenates RuBP, one 
molecule of PGA and one molecule of 2-phosphoglycolate are formed. This phosphoglycolate cannot directly enter the Calvin cycle and instead is converted to PGA via a coordinated metabolic pathway called photorespiration, which takes place in chloroplasts, peroxisomes, and mitochondria. The photorespiratory pathway involves the actions of numerous enzymes and metabolite transporters and results in the release of $\mathrm{CO}_{2}$ and ammonia and concomitant consumption of adenosine triphosphate (ATP) and reducing equivalents (Peterhansel et al., 2010).

Two different types of peroxisomal aminotransferases are active within the photorespiratory pathway, glutamate:glyoxylate aminotransferase (GGAT) and serine:glyoxylate aminotransferase (SGAT) (Igarashi et al., 2003; Liepman and Olsen, 2003). In Arabidopsis thaliana, two genes encode enzymes with GGAT activity, At1G23310 (GGT1) and At1G70580 (GGT2). These enzymes are members of aminotransferase class I, and they each catalyze transamination reactions using L-glutamate or L-alanine as amino donor and glyoxylate or pyruvate as amino acceptor (Liepman and Olsen, 2003). Plants harboring a mutation in the GGT1 gene exhibited a conditional photorespiratory phenotype of stunted growth when cultured in normal $\mathrm{CO}_{2}(0.03 \%)$; this phenotype was not evident when plants were grown in elevated $\mathrm{CO}_{2}$ (0.3\%) (Igarashi et al., 2003). The alanine:glyoxylate aminotransferase 1 (AGT1) gene (At2G13360) of Arabidopsis encodes a multifunctional class IV aminotransferase protein that catalyzes transamination reactions using L-serine, L-alanine, and L-asparagine as amino donors and glyoxylate, pyruvate, and hydroxypyruvate as amino acceptors (Liepman and Olsen, 2001; Kendziorek and Paszkowski, 2008; Zhang et al., 2013). A point mutation in the AGT1 protein (AGT1-P251L) renders sat mutant plants lethally stunted when grown in normal atmospheric conditions (Somerville and Ogren, 1980; Liepman and Olsen, 2001). Outside of photorespiration, AGT1 also has been implicated in the asparagine catabolic pathway (Ivanov et al., 2012), pathogen resistance (Taler et al., 2004), and salt tolerance (Yang et al., 2013).

Because an estimated 25\% of carbon fixed during photosynthesis is lost via photorespiration, this pathway is an appealing target for engineering efforts aiming to boost efficiency. Various approaches that may lead to such improvements are being explored, including altering the sequence of RUBISCO to improve its carboxylation rate (Whitney et al., 2011), the introduction of carbon concentration mechanisms (Lin et al., 2014a; Lin et al., 2014b), and implementation of various photorespiratory metabolic bypass pathways (Kebeish et al., 2007; Carvalho et al., 2011; Maier et al., 2012). A recent field experiment of tobacco plants engineered with a combination of bypass pathways resulted in an approximately $40 \%$ increase of biomass in transgenic lines (South et al., 2019). While these studies show great promise, it is likely that a multifaceted approach involving a combination of strategies will be needed to fine-tune photosynthesis and photorespiration for maximal efficiency and yield benefit. To inform these efforts, detailed knowledge about the structures and functions of photorespiratory proteins will be needed. Here we describe the structure of AGT1 from Arabidopsis thaliana, solved using X-ray crystallography at a resolution of $2.1 \AA$, and highlight the molecular determinants of its substrate specificity.

\section{RESULTS AND DISCUSSION}

Full-length alanine-glyoxylate aminotransferase (AGT1) from A. thaliana was expressed in Escherichia coli (Liepman and Olsen, 2001) and purified to near homogeneity using ion exchange and gel filtration chromatography. Native crystals were grown in the presence of the cofactor pyridoxal 5'-phosphate (PLP), and the structure was phased to a resolution of $3.0 \AA$ by multiple isomorphous replacement with four heavy atom derivatives using SOLVE and RESOLVE (final figure of merit =0.66). The structure, comprised of two AGT1 molecules (chain A: residues 3-401, chain B: residues 2-401) related by noncrystallographic symmetry, two PLPs, one formate, one chloride ion, and 342 water molecules, was refined to $2.2 \AA$ with PHENIX (Adams et al., 2010) (crystallographic $R_{\text {work }}=0.139$ and $R_{\text {free }}=0.170$; details in Table 1). Data were also obtained from an AGT1 crystal grown in the presence of $20 \mathrm{mM} \mathrm{L}$-serine. This isomorphous structure contained two AGT1 molecules (residues 3-401 in each), two PLPs, one hydroxypyruvate, and 422 water molecules. This structure was refined to $2.1 \AA$ with $R_{\text {work }}=0.115$ and $R_{\text {free }}=$ 0.166 . Molecules from each crystal form were almost identical; root mean square distance between $\alpha$-carbon atoms was $0.10 \AA$ for $798 \alpha$-carbons. The results described herein are for the AGT1 crystals grown in the presence of $\mathrm{L}$-serine and are valid for both structures unless explicitly mentioned.

The tertiary structure of Arabidopsis AGT1 is characteristic of the subgroup IV class of PLP-containing enzymes (Mehta et al., 1993). This group is also classified as the class $V$ aminotransferase family PF00266 by Pfam (Bateman et al., 2004). Each AGT1 subunit (Figure 1A) is comprised of a large amino-terminal domain (residues 3-269, blue in Figure 2A) containing a central 7 -stranded mixed $\beta$-sheet with 10 helices $(\mathrm{H} 1-\mathrm{H} 10)$ and a smaller carboxyl-terminal domain (270-401, green in Figure 2A) with 4 major helices (H11, H13, H15, and H16). A diagram showing the secondary structure aligned with the sequence (calculated by PDBsum; Laskowski et al., 2018) is presented in Figure 1B. Table 2 lists protein structures most similar to Arabidopsis AGT1 from a complete search (May 2019) of the Protein Data Bank (PDB; Berman et al., 2000) calculated by the Dali server (Holm and Laakso, 2016).

\section{Catalytic Dimer}

A prior study of Arabidopsis AGT1 demonstrated that the active enzyme exists as a dimer (Liepman and Olsen, 2001). Dimers were also characteristic of crystallized AGT1 (Figure 2A) and consisted of an AGT1 monomer (chain A) forming a close interface with another molecule $\left(\mathrm{A}^{\prime}\right)$ related by a crystallographic dyad along $z$. Similarly, the other molecule in the asymmetric unit, chain $\mathrm{B}$, forms a catalytic dimer with chain $\mathrm{B}^{\prime}$ related by crystallographic symmetry. Each dimer interaction buries $2,270 \AA^{2}$ of solvent-accessible surface area from each subunit (highlighted in magenta in Figure 2B), including interactions with the PLP cofactor in the respective active sites. The dimer interaction is likely essential for activity because interactions from each protomer of the dimer are needed for binding of PLP and substrate (see below). 
TABLE 1 | Crystallographic data and refinement statistics for alanine:glyoxylate aminotransferase 1 (AGT1) crystals.

\begin{tabular}{|c|c|c|}
\hline Crystal & $\begin{array}{l}\text { AGT1 native } \\
\text { PDB ID 6PK3 }\end{array}$ & $\begin{array}{c}\text { AGT with } 20 \text { mM Ser } \\
\text { PDB ID 6PK1 }\end{array}$ \\
\hline Wavelength $(\AA)$ & 1.54 & 1.54 \\
\hline Resolution range $(\AA)$ & $22.6-2.18(2.26-2.18)$ & $22.16-2.10(2.18-2.10)$ \\
\hline Space group & $P 2_{1} 2_{1} 2$ & $P 2{ }_{1} 2_{1} 2$ \\
\hline Unit cell $(\AA)$ & $\begin{array}{c}a=139.18, b=62.29 \\
c=97.01\end{array}$ & $\begin{array}{c}a=139.18, b=62.38 \\
c=96.98\end{array}$ \\
\hline No. molecules/a.u. & 2 & 2 \\
\hline Total measurements & 131,288 & 236,629 \\
\hline Unique reflections & $39,728(2,057)$ & $45,371(2,622)$ \\
\hline Multiplicity & $3.7(1.8)$ & $5.0(2.5)$ \\
\hline Completeness (\%) & 89.1 (46.8) & $90.6(53.0)$ \\
\hline Mean $/ / \sigma(l)$ & $15.4(3.5)$ & $16.0(3.8)$ \\
\hline Wilson B-factor & 23.6 & 19.62 \\
\hline R-merge & 0.077 (0.209) & $0.10(0.25)$ \\
\hline R-meas & n.a.* & $0.11(0.30)$ \\
\hline R-pim & n.a.* & $0.04(0.16)$ \\
\hline $\mathrm{CC} 1 / 2$ & n.a.* & $0.99(0.93)$ \\
\hline $\begin{array}{l}\text { Reflections used in } \\
\text { refinement }\end{array}$ & $39,723(2,058)$ & $45,359(2,624)$ \\
\hline $\begin{array}{l}\text { Reflections used for } \\
\text { R-free }\end{array}$ & $2,789(140)$ & $3,184(172)$ \\
\hline$R_{\text {cryst }}$ & $0.139(0.183)$ & $0.115(0.162)$ \\
\hline$R_{\text {free }}$ & $0.170(0.202)$ & $0.166(0.225)$ \\
\hline $\begin{array}{l}\text { Number of nonhydrogen } \\
\text { atoms }\end{array}$ & 6,650 & 6,685 \\
\hline Macromolecules & 6,272 & 6,224 \\
\hline Ligands & 36 & 39 \\
\hline Solvent & 342 & 422 \\
\hline Protein residues & 799 & 801 \\
\hline RMS (bonds) & 0.003 & 0.015 \\
\hline RMS (angles) & 0.55 & 1.13 \\
\hline $\begin{array}{l}\text { Ramachandran } \\
\text { favored (\%) }\end{array}$ & 97.4 & 96.6 \\
\hline $\begin{array}{l}\text { Ramachandran allowed } \\
(\%)\end{array}$ & 2.6 & 3.4 \\
\hline $\begin{array}{l}\text { Ramachandran outliers } \\
(\%)\end{array}$ & 0.0 & 0.0 \\
\hline Rotamer outliers (\%) & 0.59 & 0.74 \\
\hline Clashscore & 1.66 & 1.59 \\
\hline Average B-factor & 28.2 & 26.0 \\
\hline Macromolecules & 27.7 & 25.4 \\
\hline Ligands & 37.9 & 24.1 \\
\hline Solvent & 36.0 & 33.8 \\
\hline Number of TLS groups & 11 & 15 \\
\hline
\end{tabular}

*These statistics were unavailable in the version of SCALEPACK used to process the data.

Statistics for the highest-resolution shell are shown in parentheses.

Data for both crystals were collected at room temperature.

The human homolog of AGT1 (HsAGT1, PDB ID 1H0C; Zhang et al., 2003) and an AGT1 from Anabaena sp. (PDB ID 1VJO; 25\% sequence identity to AGT1; Han et al., 2005) differ in several ways from Arabidopsis AGT1. HsAGT1 and Anabaena AGT have a long amino-terminal segment ( 20 residues long) that wraps around the other subunit in an extended conformation, while this feature is absent in Arabidopsis AGT1. In Arabidopsis AGT1, the amino-terminus packs near the aminoterminal end of helix $\mathrm{H} 2$ from the other subunit (Figure 2A), and the side chain of Met4 packs against Trp327 of the same subunit. Residues 5-9 loop away from the dimer surfaces.
Unlike HsAGT1 or Anabaena AGT1, the Arabidopsis AGT1 carboxyl terminus continues with an additional turn and helix (H16, residues 374-393) that packs alongside and antiparallel to the penultimate helix $(\mathrm{H} 15,356-372)$ and helix $\mathrm{H} 11$ of the small domain (residues 271-293; Figure 2A). Helix H16 makes important noncrystallographic symmetry interactions as described in the next section. From sequence alignment of AGT1 sequence homologs, it appears that this C-terminal helix is present only among other plant AGT1 homologs, including those from melon (accession number AAL47679), lily (AAB95218), and rice (XP_015650532), and several bacterial homologs with sequence identities greater than $30 \%$ to AGT1 (e.g., Methylococcus sp. [WP_010960683], Bradyrhizobium sp. [NP_772679], and Thermotoga sp. [NP_229201]).

\section{Noncrystallographic Tetramer}

The crystallographic asymmetric unit of AGT1 contains two monomers (each from different catalytic dimers) related by a noncrystallographic two-fold axis of symmetry (NCS), which lies in the $x y$ plane, $5^{\circ}$ from the $y$-axis (molecules $\mathrm{A}$ and $\mathrm{B}$ in Figure 3A). Consequently, one catalytic dimer (A: $\left.A^{\prime}\right)$ makes close contacts with another dimer $\left(\mathrm{B}: \mathrm{B}^{\prime}\right)$ related by NCS, in effect forming an apparent "dimer of dimers" (tetramer) in the crystal (Figure 3A). Residues buried in the interface are primarily from the amino terminus, helices $\mathrm{H} 10, \mathrm{H} 13, \mathrm{H} 15, \mathrm{H} 16$, and the peroxisomal targeting sequence (PTS1) at the C-terminus (392-401) (magenta in Figure 3B). As shown in Figure 3C, the surface of the AGT1 monomer involved in the NCS interface contains nonpolar and aromatic regions (wheat and magenta surfaces, respectively) that extensively contact similar regions on the NCS-related molecule. In addition, there are $~ 30$ hydrogen bonds between molecules A and B. Each subunit of the catalytic dimer has one of these interfaces with its noncrystallographic symmetry-related mate $\left(\mathrm{A}: \mathrm{B}\right.$ as well as $\left.\mathrm{A}^{\prime}: \mathrm{B}^{\prime}\right)$. In total, about $5,200 \AA^{2}$ of the catalytic dimer solvent-accessible surface are buried in the apparent tetramer, of which about $40 \%$ is nonpolar. Analysis of the tetramer interface using PISA (Krissinel and Henrick, 2007) resulted in a complex formation significance score of 0.85 , which implies that this interface is essential for tetramer complex formation.

The apparent $M_{\mathrm{r}}$ of 92,000 , determined using size exclusion chromatography, was consistent with AGT1 being a homodimer (Liepman and Olsen, 2001). Although recombinant AGT1 is catalytically active as a dimer, it is possible that within the peroxisome, as in the crystal, it exists as a tetramer. The residues involved in the NCS interactions are conserved among putative plant orthologs of AGT1 and also some of the bacterial enzymes proposed to be serine:glyoxylate aminotransferases. Helix $\mathrm{H} 16$, which is not present in human, yeast (PDB ID 2BKW), or Anabaena AGT1, is an important component of the NCS interaction. Alternatively, these helices in AGT1 may mediate protein-protein interactions with other photorespiratory enzymes within peroxisomes, as it has been suggested that photorespiratory enzymes form complexes within the matrix of the peroxisome to efficiently channel photorespiratory metabolites and intermediates (Reumann, 2000). 

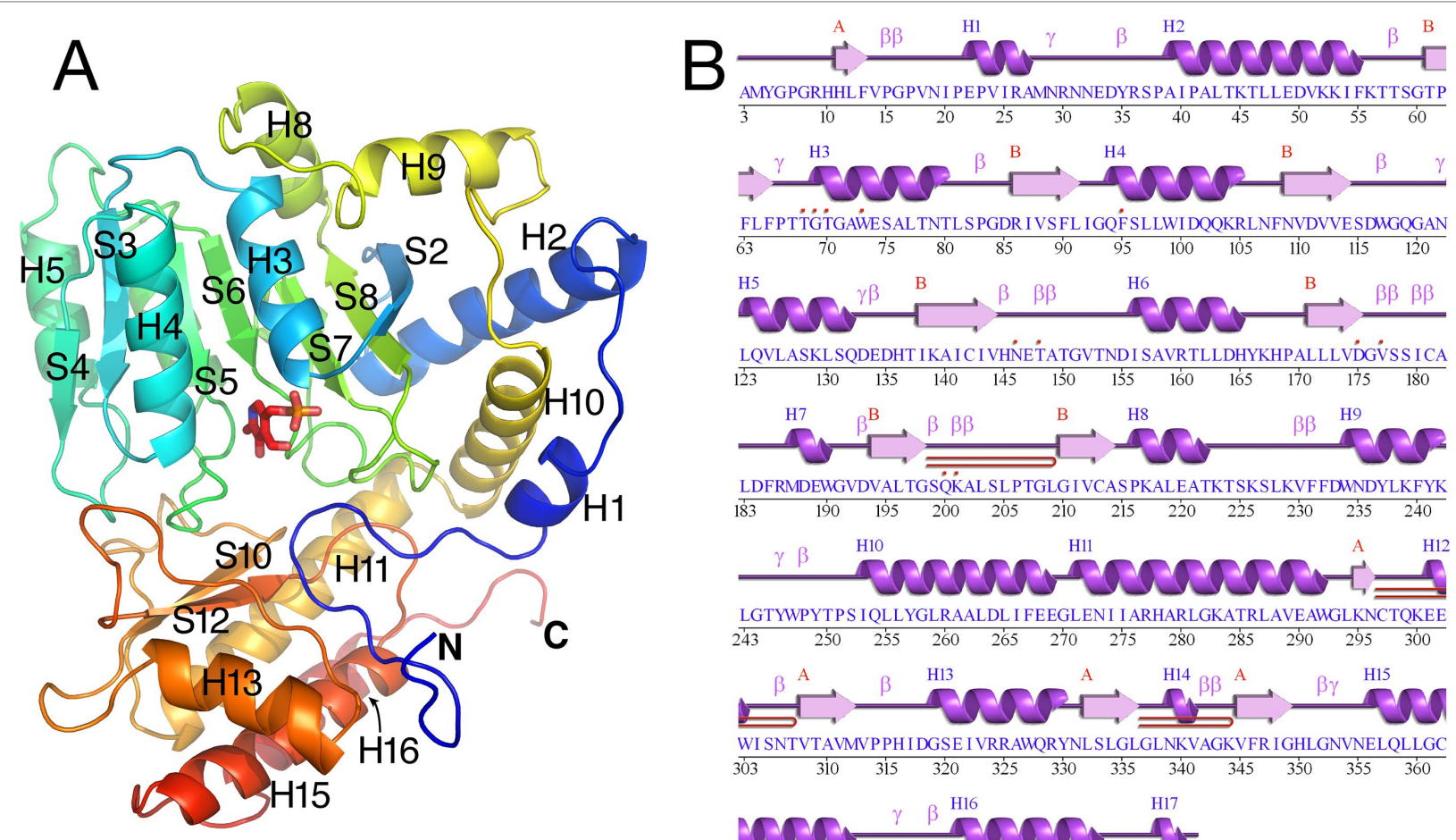
FLFPTTGǴTGAWE SALTNTL S PGDR IVSFL IGQF SLLWIDQQKRLNFNVDVVESDWGQGAN \begin{tabular}{llllllllllll}
\hline 6 & 70 & 75 & 80 & 85 & 90 & 95 & 100 & 105 & 110 & 115 & 120
\end{tabular}

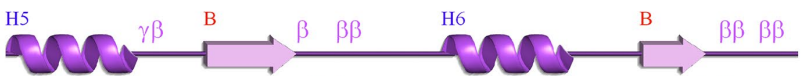

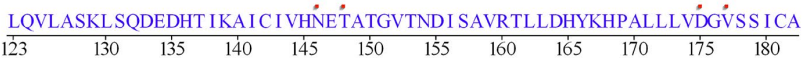

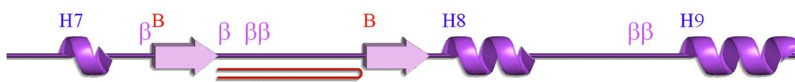
LDFRMDEWGVDVALTGSQ̉KALSLPTGLGIVCAS PKALEATKTSKSLKVFFDWNDYLKFYK

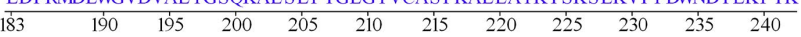

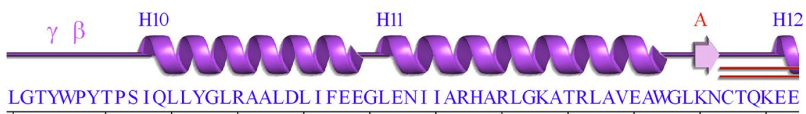

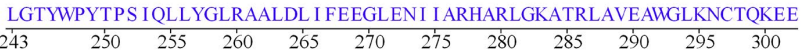

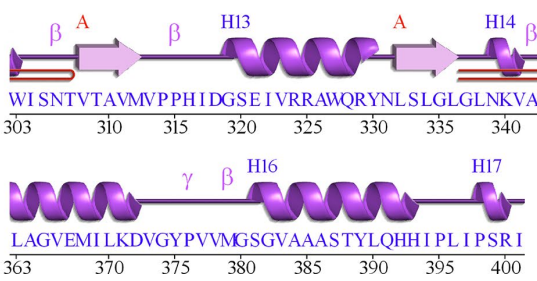

FIGURE 1 | Tertiary structure of the Arabidopsis alanine:glyoxylate aminotransferase 1 (AGT1) monomer. (A) Cartoon depiction colored from blue at the N-terminus to red at the C-terminus. Coils represent helices (labeled $\mathrm{H} n$ ); arrows represent $\beta$-strands (labeled $\mathrm{S} n$ ). The following secondary structure elements are hidden or not shown in the diagram: S1, S9, S11, H6, H7, H12, H14, and H17. (B) AGT1 primary sequence annotated with corresponding helices (Hn) and $\beta$-sheets (red letters A and B) generated by PDBsum (Laskowski et al., 2018). Turns are denoted by $\gamma$ and $\beta$. Red dots indicate residues contacting the pyridoxal $5^{\prime}$-phosphate (PLP) ligand. Hairpin turns are labeled with red lines.

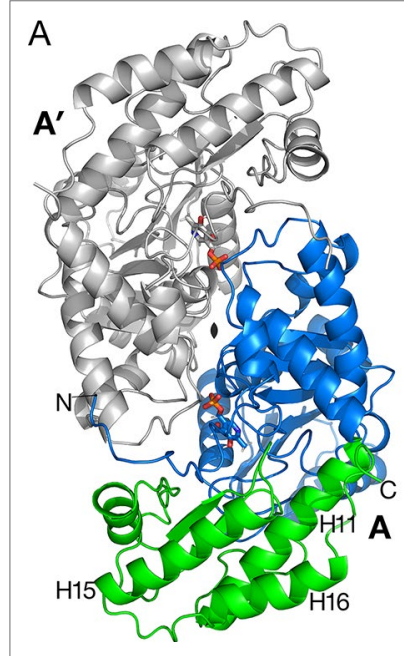

B

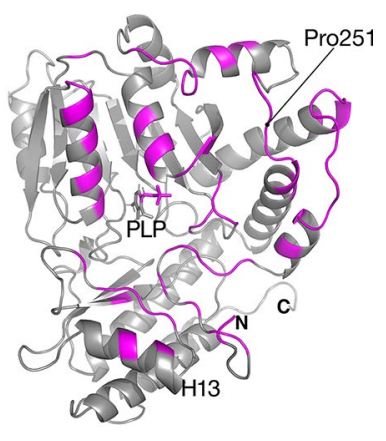

FIGURE 2 | Catalytic dimer structure. (A) Cartoon representation of subunit A with large amino-terminal domain (residues 3-269) colored blue and smaller domain (residues 270-401) colored green. The other subunit $A^{\prime}$ of the dimer (shown in gray) is related to subunit A by a two-fold crystallographic axis along $z$. Helices $\mathrm{H} 15$ and $\mathrm{H} 16$ are involved in the tetramer interactions described in Figure 3. (B) View of subunit A showing polypeptides (magenta) that are buried in the interface between $A$ and $A^{\prime}$. Residue Pro251 is the residue replaced by Leu in the sat mutant (Figure 6).

\section{Catalytic Site}

The substrate-binding site for each of the two molecules in the asymmetric unit in the native AGT1 crystal was initially refined with a PLP cofactor covalently bound to the $\varepsilon$-amino group (NZ) of Lys201, forming the expected internal aldimine structure common to most aminotransferases. Although continuous density for the NZ-C4A bond was initially present, large difference electron density suggested that the structural refinement process had artificially pulled the PLP molecule toward the Lys201 side chain and away from its correct position. Refinement of AGT1 with PLP in the aldehyde form, and without the internal aldimine bond to Lys201, resulted in a structure with little difference electron density and good geometry (Figure 4). The $\varepsilon$-amino group of Lys201 is within hydrogen bonding distance of $\mathrm{O} 3$ and O4A of PLP and the carbonyl of Pro15. A similar configuration, where no aldimine bond with the catalytic Lys was present, was observed in the AGT1 structure from Anabaena (Han et al., 2005). In Arabidopsis AGT1, the phosphate oxygens of PLP hydrogen bond to the side chains of Thr250' (prime notation refers to the residue from the other subunit of the catalytic dimer), Gln200, Thr68 and Thr70, the amide N of Gly69 and Thr70, and water (Figure 4B). The PLP ring makes nonpolar contacts with Val177 on the rear $(s i)$ face and Phe95 on the front $(r e)$ face (not shown 
TABLE 2 | Structural homologs of Arabidopsis alanine:glyoxylate aminotransferase 1 (AGT1).

\begin{tabular}{|c|c|c|c|c|}
\hline Enzyme name & Species & PDB code & $\%$ sequence identity & r.m.s.d. Å (C $\alpha$ 's aligned) \\
\hline Serine:pyruvate aminotransferase & Sulfolobus solfataricus & $3 Z R Q$ & 31 & $1.7(376)$ \\
\hline Pyridoxamine-pyruvate aminotransferase & Mesorhizobium japonicum & $2 Z 9 X$ & 26 & $1.9(385)$ \\
\hline Ureidoglycine-glyoxylate aminotransferase & Bacillus subtilis & 3ISL & 25 & $1.9(381)$ \\
\hline Ph1308 protein, serine aminotransferase & Pyrococcus horikoshii & 2DR1 & 28 & $1.8(365)$ \\
\hline Alanine:glyoxylate aminotransferase & Saccharomyces cerevisiae & 2BKW & 25 & $1.7(358)$ \\
\hline Alanine:glyoxylate aminotransferase & Anabaena sp. & $1 \mathrm{VJO}$ & 29 & $1.8(360)$ \\
\hline Alanine:glyoxylate aminotransferase & Homo sapiens & 5LUC & 27 & $1.9(365)$ \\
\hline 3-Hydroxykynurenine transaminase & Anopheles gambiae & $2 \mathrm{CH} 2$ & 25 & $2.0(367)$ \\
\hline Aminotransferase & Eubacterium rectale & $3 \mathrm{FOH}$ & 19 & $1.9(360)$ \\
\hline Alanine:glyoxylate aminotransferase & Mus musculus & 3KGW & 25 & $1.9(364)$ \\
\hline
\end{tabular}

Calculated by Dali server (http://ekhidna2.biocenter.helsinki.fi/dali) from all PDB entries (Holm and Laakso, 2016). Redundant results were omitted.

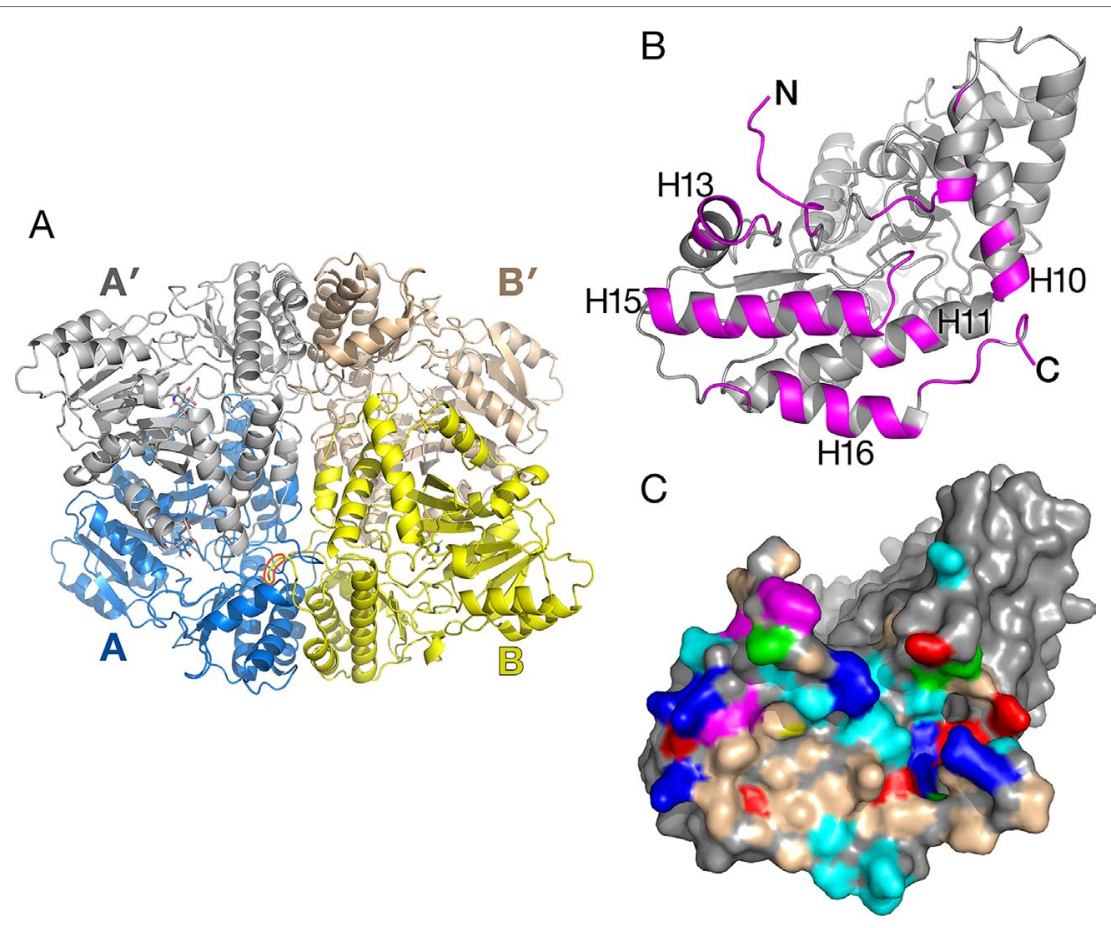

FIGURE 3 | Alanine:glyoxylate aminotransferase 1 (AGT1) tetramer present in the crystal. (A) Shown are AGT1 molecules A and B related by a noncrystallographic two-fold axis. Each forms a catalytic dimer with $\mathrm{A}^{\prime}$ and $\mathrm{B}^{\prime}$, respectively. The orange shape outlines the C-terminal peroxisomal targeting sequence (PTS) of molecule B where it interacts with molecule A. (B) Molecule A is rotated about $90^{\circ}$ to show the surface that forms the interface with molecule B. Magenta-colored polypeptides are buried in the interface. (C) Surface representation of molecule A [same orientation as (B)]. Colored surface represents residues that form the interface with molecule B. Nonpolar regions (wheat-colored) and aromatic residues (magenta-colored) contribute to the hydrophobic interactions in the tetramer interface. Other residue colors: blue, positively charged; red, negatively charged; cyan, polar; green, proline.

in Figure 4B) and Trp73 at the bottom. The conserved Asp175 forms a hydrogen bond to the PLP ring N1 at the bottom of the ring and Thr148 forms a hydrogen bond to O1 of the PLP.

In the AGT1 structure crystallized in the presence of L-serine, a pyridoxamine phosphate (PMP) molecule and no internal aldimine bond were expected, but the geometry and close distance of the PMP amino group (N4A) to the Lys201 NZ suggested that the cofactor should best be modeled as PLP.

In the substrate binding site of the AGT1 crystal grown in the presence of L-serine, there was positive difference electron density suggestive of a bound molecule near the guanidinium group of Arg347 in chain A (Figure 5A). This density, in analogy with known structures of aminotransferase:substrate complexes, is directly above the PLP and is a likely location for the L-serine substrate or hydroxypyruvate product (ligand 3PY). The latter could be refined at partial occupancy, but there was no electron density for the hydroxyl group in the resulting map (Supplementary Figure 1). Using this ligand as a guide, we modeled an L-serine in the same position (Figure $5 \mathbf{B}$ ). Surrounding the putative ligand (in clockwise order) are Thr250', Tyr35', Arg36', Gly16, Pro15, Arg347, and Thr148. At the bottom of the site are PLP and Lys201. The side chain hydroxyl of the 

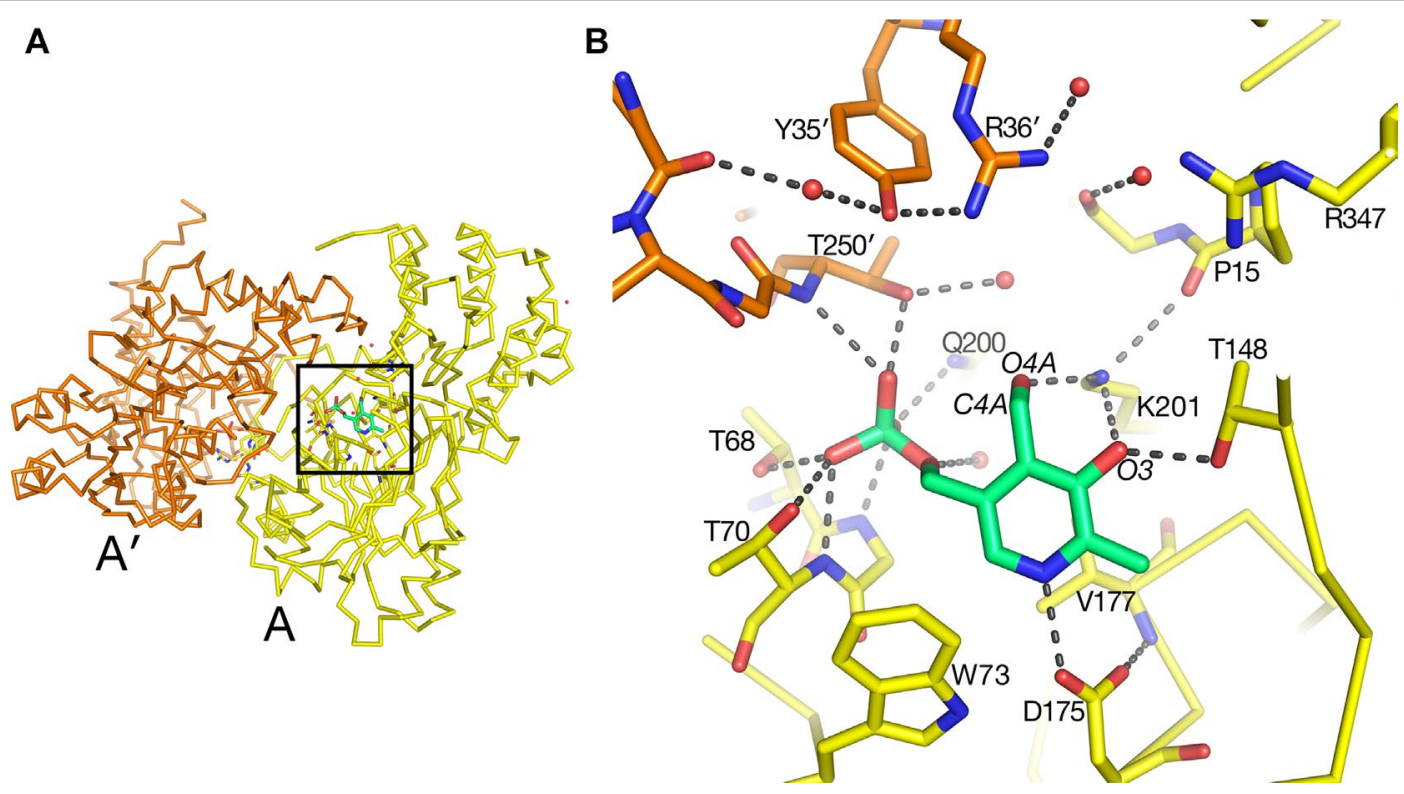

FIGURE 4 | Pyridoxal 5'-phosphate (PLP)-binding site in alanine:glyoxylate aminotransferase 1 (AGT1). (A) Entire AGT1 dimer oriented as in the blow-up in (B). Box outlines the portion of the structure depicted in (B). (B) Yellow carbon sticks are from molecule A; orange carbon sticks are from the other subunit in the dimer $\left(A^{\prime}\right)$; green carbon and phosphate sticks are the PLP molecule bound to subunit A. Small red spheres represent water molecules. Residues are labeled with their one-letter code.

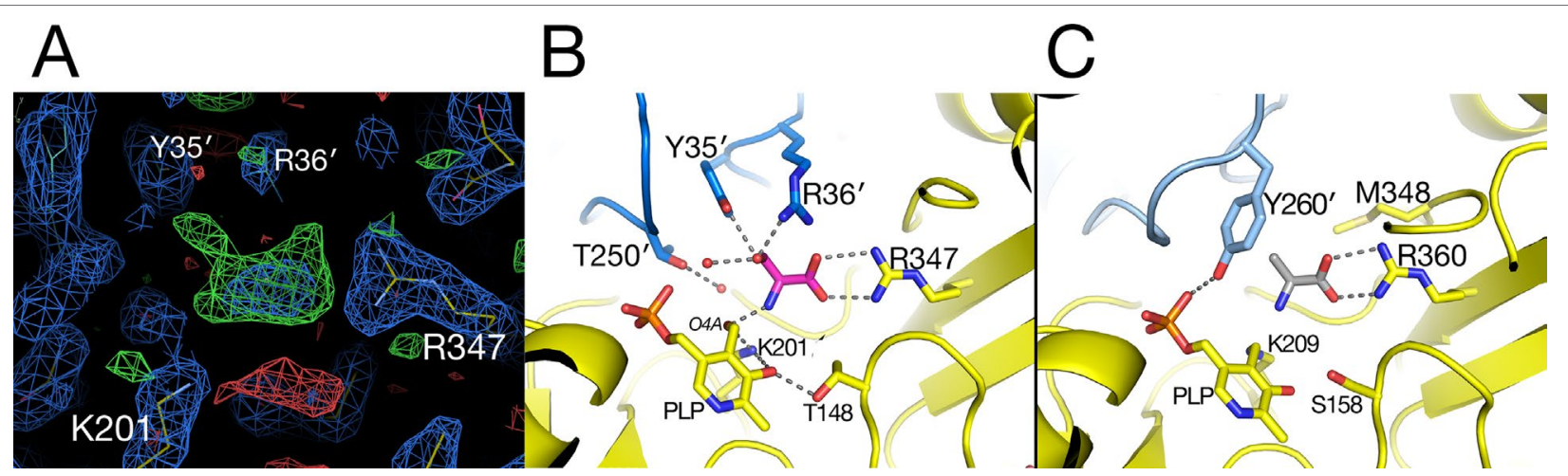

FIGURE 5 | Substrate-binding site in alanine:glyoxylate aminotransferase 1 (AGT1). (A) Electron density near Arg347 suggestive of a bound substrate. Blue contours are from a $2 m F_{\text {obs }}-D F_{\text {calc }}$ electron density map contoured in Coot at 1.2 root mean square deviations above the mean. Green contours are a $m F_{\text {obs }}{ }^{-}$ $D F_{\text {calc }}$ difference electron density map contoured in Coot at 2.25 times the root mean square deviation above the mean. Hydroxypyruvate was modeled into the electron density and refined to an occupancy of 0.67 , yet no density was observed for the hydroxymethyl group (see Supplementary Figure 1). (B) L-Serine molecule (magenta) was modeled in AGT1 guided by the difference density in (A). Residues with yellow carbons are from subunit A, and those with blue carbons are from the dimer's other subunit ( $\mathrm{A}^{\prime}$ ). The L-serine hydroxyl group is within hydrogen-bonding distance of Tyr35', Arg36', and a water molecule. (C) Substrate binding site of human AGT1 (PDB ID 1HOC) viewed in the same orientation as (B) but with an L-alanine (gray carbons) modeled in place of serine. The Tyr260' aromatic ring may sterically clash with the hydroxyl of L-serine.

modeled L-serine is within hydrogen bonding distance of both Tyr35' and Arg36' from the other subunit of the dimer. Arg347 is found in all aminotransferases and forms ionic hydrogen bonds to the carboxylate of the modeled serine. The L-serine amino group is oriented toward the $\varepsilon$-amino group of the catalytic Lys201 and the aldehyde O4A of PLP. In the structure of native Arabidopsis AGT1, smaller difference electron density near Arg347 was modeled in chain A as a formate molecule, which was present in high concentration during crystallization, or as a chloride ion in chain B.

When the active site region of the refined structure of Arabidopsis AGT1 is compared to the human AGT1 (HsAGT1) structure (which prefers L-alanine as substrate), the most significant differences are in the region of Arabidopsis AGT1 residues Tyr35' and Arg36' (Figure 5B). In HsAGT1, the structurally equivalent residues are Ser48' and Met49', but 
these are too distant from the active site to interact with substrate. Instead, Tyr260' of HsAGT1 enters the substrate binding site and the aromatic ring forms a nonpolar wall; the Tyr hydroxyl group hydrogen bonds to the phosphate and is not available for substrate interaction (Figure 5C). Lower catalytic activity of HsAGT1 with the substrate L-serine (Okuno et al., 1980) could be due to steric interactions of the serine hydroxyl with the Tyr260' ring. The AGT1 homolog from Anabaena has an active site structure similar to that in HsAGT1 and also contains a conserved tyrosine, suggesting that it also may prefer L-alanine over L-serine as an amino donor. Although the enzyme from Sulfolobus solfataricus, with $31 \%$ sequence identity and structural similarity to Arabidopsis AGT1, is named a serine:pyruvate aminotransferase (PDB ID 3ZRQ), phenylalanine and other hydrophobic amino donors are actually much better substrates than serine (Sayer et al., 2012). This is consistent with the configuration of its active site, which is more similar to HsAGT1 than to Arabidopsis AGT1.

In $E$. coli phosphoserine aminotransferase (PDB ID 1BJO with $13 \%$ sequence identity to AGT1), another group IV aminotransferase, residues His41' and Arg42' occupy similar positions as Tyr35' and Arg36' of Arabidopsis AGT1 and interact with the carboxylate side chain of a substrate analog (Hester et al., 1999). Together, these results suggest that Tyr35' and Arg36' may enable Arabidopsis AGT1 to use L-serine as an amino donor substrate. Indeed, these two residues are highly conserved among a wide range of known and putative plant serine:glyoxylate amino transferases. Although not modeled here, a report indicating that L-asparagine also acts as an amino donor substrate of Arabidopsis AGT1 (Ivanov et al., 2012) could also be consistent with the Arabidopsis AGT1 active site configuration shown in Figure 5B.

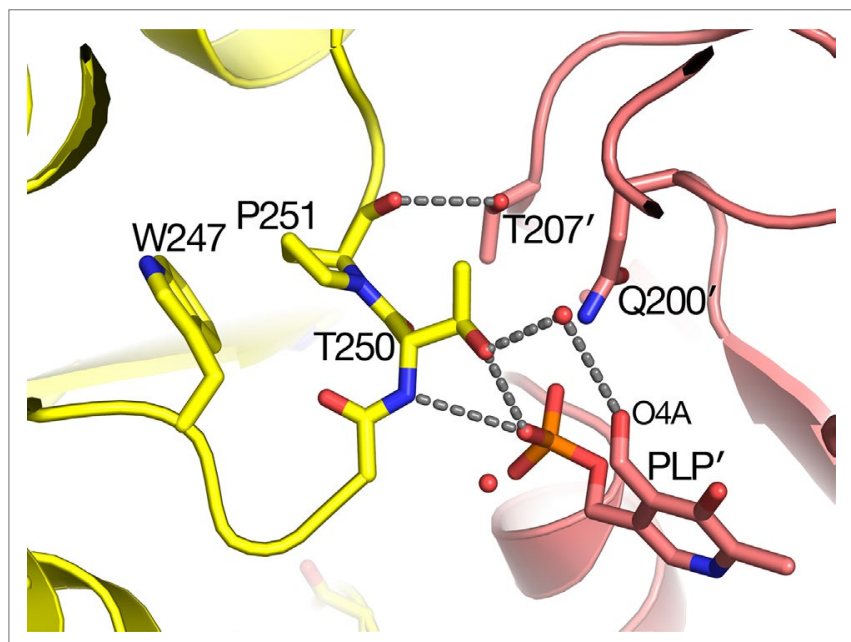

FIGURE 6 | Substitution of Pro251 by Leu in the sat mutant results in an inactive enzyme. Carbon atoms of molecule $A$ are colored yellow, while rosecolored atoms are from the other subunit $\left(\mathrm{A}^{\prime}\right)$ in the catalytic dimer. Pro251 and the adjacent Thr250 make interactions with the other subunit including pyridoxal 5'-phosphate PLP'). It is likely that the Leu substitution at residue 251 would distort the backbone conformation of 250-251 because it would sterically clash with the nearby Trp247.

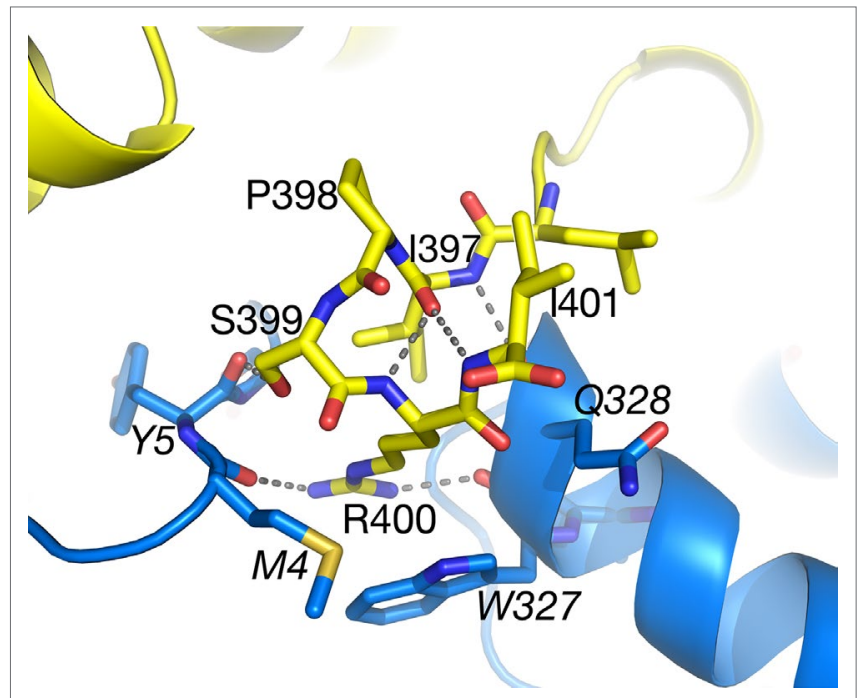

FIGURE 7 | The C-terminal peroxisomal targeting sequence (PTS) sequence (Ser399-Ile401) of subunit A (yellow carbons) lies on the surface of the tetramer at the interface with subunit B (blue carbons). Also see Figure 3A. lle401 is totally accessible to solvent. Italicized labels denote residues from subunit $B$

\section{The sat Mutant Is at the Dimer Interface}

The sat mutant of Arabidopsis thaliana exhibits a photorespiratory air-sensitive phenotype (Somerville and Ogren, 1980) due to a point mutation that causes a substitution of Leu for Pro at position 251 of Arabidopsis AGT1 (Liepman and Olsen, 2001). The structure provides additional insights about the mechanistic consequences of the P251L mutation in Arabidopsis AGT1. Pro251 is found on an extended loop just prior to helix $\mathrm{H} 10$ of the larger domain (Figure 2B). This loop is at the dimer interface and close to the active site and $\mathrm{PLP}^{\prime}$ of the other subunit (Figure 6). The Pro251 O hydrogen bonds to the Thr207' hydroxyl. The adjacent side chain of Thr250 also forms hydrogen bonds to the nearby PLP' $^{\prime}$ phosphate and to an active site water molecule, which hydrogen bonds to the PLP' aldehyde (O4A). Because the Pro251 side chain packs against the ring of $\operatorname{Tr} 2247$, replacement with leucine would likely distort the position of the loop and disrupt the hydrogen-bonds to the waters and phosphate of the nearby active site. It may also affect dimerization, rendering the enzyme inactive.

\section{Peroxisomal Targeting Sequence Resolved}

Within the structure of Arabidopsis AGT1, the carboxyl-terminal tripeptide Ser-Arg-Ile (residues 399-401) constituting a type 1 peroxisomal targeting sequence (PTS1; Chowdhary et al., 2012) was well resolved in the electron density. In the context of the monomer or catalytic dimer, the PTS1 residues are exposed to solvent as one would expect for the in vivo recognition by the peroxisomal protein import machinery. However, in the tetramer interface (Figure 7), the side chains of residues 399-400 are on the surface but make interactions with the NCS-related chain B (carbons in blue). The PTS1 forms one turn of an a-helix facilitated by hydrogen bonds 
from the carbonyl of Ile397. The Ser399 hydroxyl forms a hydrogen bond to the carbonyl of residue 5 from the NCS-related molecule (chain B) and a water in the interface. Arg400 packs between residues 4 and 5 and residues 327-329 of chain B and forms hydrogen bonds to chain B residues Met 4 O, Trp327 O, and Asn331 OD1. Only Ile401 is fully solvent exposed. These interactions would make it unlikely for the tetramer to exist in the cytoplasm where the PTS1 must be recognized by its receptor. In contrast, the PTS1 in the HsAGT1 dimer structure is also solvent exposed but not resolved in the electron density map. When HsAGT1 is in complex with its peroxisomal receptor Pex5p, the PTS1 sequence (Lys-LysLeu) exists in an extended conformation with extensive interactions with Pex5p (PDB ID 3R9A; Fodor et al., 2012).

\section{Future Directions}

The reaction mechanism of human AGT1 has been described in detail (Giardina et al., 2017), and because the catalytic residues are conserved in Arabidopsis AGT1, this protein likely has the same mechanism. Recent structures of human AGT1 (Giardina et al., 2017) show evidence of X-ray radiation-induced reduction and breakage of the internal aldimine bond between PLP C4' and Lys209 $\mathrm{Ne}$ (equivalent to Lys201 in Arabidopsis AGT1), together with a change in tilt of the PLP ring to optimize the hydrogen bond between Asp183 (Asp175 in Arabidopsis AGT1) and PLP N1 (Figure 4). It is possible that radiation damage also caused the lack of the internal aldimine bond observed in the Arabidopsis AGT1 structure since data collection time was on the order of hours at room temperature. The internal aldimine bond also was not observed in the structure of AGT from Anabaena, even though data used for this structure were collected using cryo-cooled crystals with a synchrotron (Han et al., 2005). Future structural analyses using shorter exposures with an appropriate radical scavenger in the cryosolvent may reveal whether Arabidopsis AGT1 possesses an intact internal aldimine bond with PLP and provide additional data that could enable computational studies of the enzyme mechanism.

\section{METHODS}

\section{Purification Of Recombinant AGT1}

Untagged recombinant AGT1 from Arabidopsis thaliana (AGI: At2G13360) was expressed in E. coli BL21(DE3) cells containing an expression plasmid derived from pET28a (Novagen) as previously described (Liepman and Olsen, 2001). For crystallization experiments, AGT1 was purified using a two-step purification procedure that exploits the affinity of AGT1 for its cofactor PLP (Hondred et al., 1985). PLP was bound to a Q-Sepharose FF (Pharmacia) anion exchange column prior to application of the crude soluble fraction of the cell lysate. AGT1 was eluted from the column using a linear gradient of $\mathrm{KCl}(0-250 \mathrm{mM})$ in buffer containing $50 \mathrm{mM}$ Tris-HCl, $0.2 \mathrm{mM}$ PLP, and $10 \%$ glycerol ( $\mathrm{pH}$ 8.5). Peak fractions identified using SDS-PAGE and activity assays (Liepman and Olsen, 2001) were pooled and concentrated using an Amicon stirred ultrafiltration unit with a membrane MWCO of 10,000. The concentrated sample was applied to a Superose 6 gel filtration column (Pharmacia) pre-equilibrated with buffer containing 50 $\mathrm{mM}$ Tris- $\mathrm{HCl}, 0.2 \mathrm{mM}$ PLP, $10 \%$ glycerol, and $50 \mathrm{mM} \mathrm{NaCl}(\mathrm{pH}$ 8.5). Peak gel filtration fractions were pooled and concentrated to 11 $\mathrm{mg} / \mathrm{ml}$ using an Amicon stirred ultrafiltration cell. Supplementary Figure 2 shows SDS-PAGE analysis of the purified protein.

\section{Crystallization of AGT1}

AGT1 was screened for crystallization by the microvapor diffusion technique using precipitants from Crystal Screen I (Hampton Research). Crystals with different morphologies appeared after several days in several of the precipitant conditions. After optimization, large, greenish-yellow crystals were grown from drops containing equal volumes of protein $(11 \mathrm{mg} / \mathrm{ml}$ in $0.2 \mathrm{mM}$ PLP, $10 \%$ glycerol, $100 \mathrm{mM}$ Tris$\mathrm{HCl} \mathrm{pH} \mathrm{8.5)} \mathrm{and} \mathrm{precipitant} \mathrm{(4.1-4.2} \mathrm{M} \mathrm{sodium} \mathrm{formate)}$ equilibrated against the same precipitant at room temperature (Supplementary Figure 2). Crystals grown in the same buffer containing $20 \mathrm{mM} \mathrm{L}$-serine grew more consistently and were clear. Typical crystals grew to $\sim 0.5 \times 0.3 \times 0.2 \mathrm{~mm}$ within a week.

\section{MIR Phasing and Structure Refinement}

Since initial attempts to flash-cool the crystals were unsuccessful, all data sets were collected at $25^{\circ} \mathrm{C}$ in capillary mounts. One-degree oscillation images (exposure $\approx 5 \mathrm{~min}$ ) were collected on a Rigaku/MSC R-AXIS IV detector/rotating anode generator $(100 \mathrm{~mA}, 48 \mathrm{kV})$. Reflections were indexed, integrated, and scaled with the HKL package (Otwinowski and Minor, 1997). Native AGT1 crystals typically diffracted to at least $2.4 \AA$ resolution, and some up to $2.2 \AA$. AGT1 crystals were orthorhombic, in space group $P 22_{1} 22$, and with unit cell parameters $a=140.2 \AA, b=62.8 \AA, c=98.2 \AA$. Two molecules were present in the asymmetric unit.

Native crystals grown in the presence of PLP but without L-serine were colored and expected to be in the internal aldimine form. Diffraction data statistics for such crystals are shown in Table 1. Unmerged reflections were not available for the native data, so $\mathrm{CC} 1 / 2$ values could not be calculated. Data (not shown) were also collected from four heavy-atom derivative crystals: mercury chloride, gold cyanide, potassium tetrachloroplatinate, and $p$-hydroxymercuribenzoate. Data to $2.1 \AA$ resolution were collected from the crystals grown in 20 mM L-serine.

MIR phases were calculated by the SOLVE program (version 2.03) (Terwilliger, 2003) with four heavy atom derivative data sets. The final mean figure of merit $\langle m\rangle$ was 0.44 for 16,917 reflections from 20.0 to $3.0 \AA$. Density modification by RESOLVE (Terwilliger, 2003) raised the $\langle m\rangle$ to 0.66 with a mean phase change of $44^{\circ}$ from the MIR phases. A model of AGT1 containing $~ 84 \%$ of the amino acid residues was constructed into the resulting $3.0 \AA$. This model was transformed to generate the other molecule in the asymmetric unit, and its orientation was optimized by real-space rigid body minimization. The remainder of the structure was built with Coot (Emsley et al., 2010) and refined with CNS (Brünger et al., 1998) with noncrystallographic symmetry 
restraints between the two molecules in the asymmetric unit. The structure was further refined without NCS restraints in Phenix to 2.2- $\AA$ resolution to a $R_{\text {cryst }}$ of $0.139\left(R_{\text {free }}=0.170\right)$ and contains two AGT1 molecules, two PLPs, one formate, one chloride, and 342 water molecules (Table 1). The structure from crystals grown in the presence of L-serine was refined with Phenix to $2.1 \AA$ resolution with an $R_{\text {cryst }} / R_{\text {free }}$ of 0.115/0.166 and contains two AGT1 molecules, two PLPs, one hydroxypyruvate, and 422 water molecules. Coordinate files and structure factors have been deposited in the Protein Data Bank as entries 6PK3 (native AGT1) and 6PK1 (AGT1 grown in presence of $20 \mathrm{mM}$ Ser).

Interface and solvent accessibility calculations were done with the PISA server (https://www.ebi.ac.uk/pdbe/pisa/). All figures were prepared with PyMOL version 2.2 (Schrödinger LLC).

\section{DATA AVAILABILITY STATEMENT}

The datasets generated for this study can be found in the Protein Data Bank (www.rcsb.org), Accession IDs: AGT1 native, 6PK3; AGT1 with serine, 6PK1.

\section{REFERENCES}

Adams, P. D., Afonine, P. V., Bunkóczi, G., Chen, V. B., Davis, I. W., Echols, N., et al. (2010). PHENIX: a comprehensive Python-based system for macromolecular structure solution. Acta Crystallogr. D Biol. Crystallogr. 66, 213-221. doi: 10.1107/S0907444909052925

Bateman, A., Coin, L., Durbin, R., Finn, R. D., Hollich, V., Griffiths-Jones, S., et al. (2004). The Pfam protein families database. Nucleic Acids Res. 32, D138-D141. doi: 10.1093/nar/gkh121

Berman, H. M., Westbrook, J., Feng, Z., Gilliland, G., Bhat, T. N., Weissig, H., et al. (2000). The Protein Data Bank. Nucleic Acids Res. 28, 235-242. doi: 10.1093/ nar/28.1.235

Brünger, A. T., Adams, P. D., Clore, G. M., DeLano, W. L., Gros, P., GrosseKunstleve, R. W., et al. (1998). Crystallography \& NMR system: a new software suite for macromolecular structure determination. Acta Crystallogr. D Biol. Crystallogr. 54, 905-921. doi: 10.1107/S0907444998003254

Carvalho, J. F. C., Madgwick, P. J., Powers, S. J., Keys, A. J., Lea, P. J., and Parry, M. A. (2011). An engineered pathway for glyoxylate metabolism in tobacco plants aimed to avoid the release of ammonia in photorespiration. BMC Biotechnol. 11, 111. doi: 10.1186/1472-6750-11-111

Chowdhary, G., Kataya, A. R., Lingner, T., and Reumann, S. (2012). Non-canonical peroxisome targeting signals: identification of novel PTS1 tripeptides and characterization of enhancer elements by computational permutation analysis. BMC Plant Biol. 12, 142. doi: 10.1186/1471-2229-12-142

Emsley, P., Lohkamp, B., Scott, W. G., and Cowtan, K. (2010). Features and development of Coot. Acta Crystallogr. D Biol. Crystallogr. 66, 486-501. doi: 10.1107/S0907444910007493

Fodor, K., Wolf, J., Erdmann, R., Schliebs, W., and Wilmanns, M. (2012). Molecular requirements for peroxisomal targeting of alanine-glyoxylate aminotransferase as an essential determinant in primary hyperoxaluria type 1. PLoS Biol. 10, e1001309. doi: 10.1371/journal.pbio.1001309

Giardina, G., Paiardini, A., Montioli, R., Cellini, B., Voltattorni, C. B., and Cutruzzolà, F. (2017). Radiation damage at the active site of human alanine:glyoxylate aminotransferase reveals that the cofactor position is finely tuned during catalysis. Sci. Rep. 7, 11704. doi: 10.1038/ s41598-017-11948-w

Han, G. W., Schwarzenbacher, R., Page, R., Jaroszewski, L., Abdubek, P., Ambing, E., et al. (2005). Crystal structure of an alanine-glyoxylate aminotransferase from

\section{AUTHOR CONTRIBUTIONS}

$\mathrm{AL}$ and $\mathrm{BH}$ purified AGT1 protein; AL crystallized the protein, $\mathrm{AL}, \mathrm{JV}, \mathrm{DP}$, and $\mathrm{BH}$ collected diffraction data; JV calculated phases; JV and MS refined the structure. AL and MS wrote the manuscript and made the figures; AL, JV, DP, BH, LO, and MS edited the manuscript.

\section{ACKNOWLEDGMENTS}

This work was supported, in part, by fellowships to AL from the University of Michigan Cellular Biotechnology Training Program (NIH-GM08353) and the University of Michigan Rackham School of Graduate Studies and a Faculty Research Fellowship Award from Eastern Michigan University.

\section{SUPPLEMENTARY MATERIAL}

The Supplementary Material for this article can be found online at: https://www.frontiersin.org/articles/10.3389/fpls.2019.01229/ full\#supplementary-material.

Anabaena sp. at $1.70 \AA$ A resolution reveals a noncovalently linked PLP cofactor. Proteins 58, 971-975. doi: 10.1002/prot.20360

Hester, G., Stark, W., Moser, M., Kallen, J., Markovic-Housley, Z., and Jansonius, J. N. (1999). Crystal structure of phosphoserine aminotransferase from Escherichia coli at $2.3 \AA$ resolution: comparison of the unligated enzyme and a complex with alpha-methyl-1-glutamate. J. Mol. Biol. 286, 829-850. doi: 10.1006/jmbi.1998.2506

Holm, L., and Laakso, L. M. (2016). Dali server update. Nucleic Acids Res. 44, W351-W355. doi: 10.1093/nar/gkw357

Hondred, D., Hunter, J. M., Keith, R., Titus, D. E., and Becker, W. M. (1985). Isolation of serine:glyoxylate aminotransferase from cucumber cotyledons. Plant Physiol. 79, 95-102. doi: 10.1104/pp.79.1.95

Igarashi, D., Miwa, T., Seki, M., Kobayashi, M., Kato, T., Tabata, S., et al. (2003). Identification of photorespiratory glutamate:glyoxylate aminotransferase (GGAT) gene in. Arabidopsis Plant J. 33, 975-987. doi: 10.1046/j.1365-313X.2003.01688.x

Ivanov, A., Kameka, A., Pajak, A., Bruneau, L., Beyaert, R., Hernández-Sebastià, C., et al. (2012). Arabidopsis mutants lacking asparaginases develop normally but exhibit enhanced root inhibition by exogenous asparagine. Amino Acids 42, 2307-2318. doi: 10.1007/s00726-011-0973-4

Kebeish, R., Niessen, M., Thiruveedhi, K., Bari, R., Hirsch, H.-J., Rosenkranz, R., et al. (2007). Chloroplastic photorespiratory bypass increases photosynthesis and biomass production in. Arabidopsis thaliana Nat. Biotechnol. 25, 593-599. doi: $10.1038 /$ nbt1299

Kendziorek, M., and Paszkowski, A. (2008). Properties of serine:glyoxylate aminotransferase purified from Arabidopsis thaliana leaves. Acta Bioch. Bioph. Sin. 40, 102-110. doi: 10.1111/j.1745-7270.2008.00383.x

Krissinel, E., and Henrick, K. (2007). Inference of macromolecular assemblies from crystalline state. J. Mol. Biol. 372, 774-797. doi: 10.1016/j.jmb.2007.05.022

Laskowski, R. A., Jabłońska, J., Pravda, L., Vařeková, R. S., and Thornton, J. M. (2018). PDBsum: Structural summaries of PDB entries. Protein Sci. 27, 129134. doi: 10.1002/pro.3289

Liepman, A. H., and Olsen, L. J. (2003). Alanine aminotransferase homologs catalyze the glutamate:glyoxylate aminotransferase reaction in peroxisomes of Arabidopsis. Plant Physiol. 131, 215-227. doi: 10.1104/pp.011460

Liepman, A. H., and Olsen, L. J. (2001). Peroxisomal alanine: glyoxylate aminotransferase (AGT1) is a photorespiratory enzyme with multiple substrates in. Arabidopsis thaliana Plant J. 25, 487-498. doi: 10.1046/j.1365-313x.2001.00961.x 
Lin, M. T., Occhialini, A., Andralojc, P. J., Devonshire, J., Hines, K. M., Parry, M. A. J., et al. (2014a). $\beta$-Carboxysomal proteins assemble into highly organized structures in Nicotiana chloroplasts. Plant J. 79, 1-12. doi: 10.1111/tpj.12536

Lin, M. T., Occhialini, A., Andralojc, P. J., Parry, M. A. J., and Hanson, M. R. (2014b). A faster Rubisco with potential to increase photosynthesis in crops. Nature 513, 547-550. doi: 10.1038/nature13776

Maier, A., Fahnenstich, H., von Caemmerer, S., Engqvist, M. K. M., Weber, A. P. M., Flügge, U., et al. (2012). Transgenic introduction of a glycolate oxidative cycle into A. thaliana chloroplasts leads to growth improvement. Front. Plant Sci. 3, 38. doi: 10.3389/fpls.2012.00038

Mehta, P. K., Hale, T. I., and Christen, P. (1993). Aminotransferases: demonstration of homology and division into evolutionary subgroups. Eur. J. Biochem. 214, 549-561. doi: 10.1111/j.1432-1033.1993.tb17953.x

Okuno, E., Minatogawa, Y., Nakamura, M., Kamoda, N., Nakanishi, J., Makino, M., et al. (1980). Crystallization and characterization of human liver kynurenineglyoxylate aminotransferase. Identity with alanine-glyoxylate aminotransferase and serine-pyruvate aminotransferase. Biochem. J. 189, 581-590. doi: 10.1042/ bj1890581

Otwinowski, Z., and Minor, W. (1997). Processing of X-ray diffraction data collected in oscillation mode. Meth. Enzymol. 276, 307-326. doi: 10.1016/ S0076-6879(97)76066-X

Peterhansel, C., Horst, I., Niessen, M., Blume, C., Kebeish, R., Kürkcüoglu, S., et al. (2010). Photorespiration. Arabidopsis Book 8, e0130. doi: 10.1199/tab.0130

Reumann, S. (2000). The structural properties of plant peroxisomes and their metabolic significance. Biol. Chem. 381, 639-648. doi: 10.1515/BC.2000.084

Sayer, C., Bommer, M., Isupov, M., Ward, J., and Littlechild, J. (2012). Crystal structure and substrate specificity of the thermophilic serine:pyruvate aminotransferase from Sulfolobus solfataricus. Acta Crystallogr. D Biol. Crystallogr. 68, 763-772. doi: 10.1107/S0907444912011274

Sharkey, T. D. (1988). Estimating the rate of photorespiration in leaves. Physiol. Plant. 73, 147-152. doi: 10.1111/j.1399-3054.1988.tb09205.x

Somerville, C. R., and Ogren, W. L. (1980). Photorespiration mutants of Arabidopsis thaliana deficient in serine-glyoxylate aminotransferase activity. Proc. Natl. Acad. Sci. U. S. A. 77, 2684-2687. doi: 10.1073/pnas.77.5.2684

South, P. F., Cavanagh, A. P., Liu, H. W., and Ort, D. R. (2019). Synthetic glycolate metabolism pathways stimulate crop growth and productivity in the field. Science 363, eaat9077. doi: 10.1126/science.aat9077
Taler, D., Galperin, M., Benjamin, I., Cohen, Y., and Kenigsbuch, D. (2004). Plant $e R$ genes that encode photorespiratory enzymes confer resistance against disease. Plant Cell 16, 172-184. doi: 10.1105/tpc.016352

Terwilliger, T. C. (2003). SOLVE and RESOLVE: automated structure solution and density modification. Meth. Enzymol. 374, 22-37. doi: 10.1016/ S0076-6879(03)74002-6

Whitney, S. M., Sharwood, R. E., Orr, D., White, S. J., Alonso, H., and Galmes, J. (2011). Isoleucine 309 acts as a C4 catalytic switch that increases ribulose1,5-bisphosphate carboxylase/oxygenase (rubisco) carboxylation rate in Flaveria. Proc. Natl. Acad. Sci. U. S. A. 108, 14688-14693. doi: 10.1073/ pnas. 1109503108

Yang, L., Han, H., Liu, M., Zuo, Z., Zhou, K., Lü, J., et al. (2013). Overexpression of the Arabidopsis photorespiratory pathway gene, serine: glyoxylate aminotransferase (AtAGT1), leads to salt stress tolerance in transgenic duckweed (Lemna minor). Plant Cell Tiss. Organ Cult. 113, 407-416. doi: 10.1007/s11240-012-0280-0

Zhang, Q., Lee, J., Pandurangan, S., Clarke, M., Pajak, A., and Marsolais, F. (2013). Characterization of Arabidopsis serine:glyoxylate aminotransferase, AGT1, as an asparagine aminotransferase. Phytochemistry 85, 30-35. doi: 10.1016/j. phytochem.2012.09.017

Zhang, X., Roe, S. M., Hou, Y., Bartlam, M., Rao, Z., Pearl, L. H., et al. (2003). Crystal structure of alanine:glyoxylate aminotransferase and the relationship between genotype and enzymatic phenotype in primary hyperoxaluria type 1. J. Mol. Biol. 331, 643-652. doi: 10.1016/ S0022-2836(03)00791-5

Conflict of Interest: The authors declare that the research was conducted in the absence of any commercial or financial relationships that could be construed as a potential conflict of interest.

Copyright (C) 2019 Liepman, Vijayalakshmi, Peisach, Hulsebus, Olsen and Saper. This is an open-access article distributed under the terms of the Creative Commons Attribution License (CC BY). The use, distribution or reproduction in other forums is permitted, provided the original author(s) and the copyright owner(s) are credited and that the original publication in this journal is cited, in accordance with accepted academic practice. No use, distribution or reproduction is permitted which does not comply with these terms. 